\title{
IMPORTANCIA Y COINCIDENCIA DE COMPETENCIAS PARA EGRESADOS DE ADMINISTRACIÓN Y EMPLEADORES: UN ENFOQUE POR REGIONES DE COLOMBIA*
}

\author{
LEONOR CABEZA**, JAIME CASTRILLÓN**** \& JAHIR LOMBANA***** \\ UNIVERSIDAD DEL NORTE
}

Recibido/Received/Recebido: 08/07/2016 - Aceptado/ Accepted/Aprovado: 22/08/2016

\begin{abstract}
Resumen
Este documento se fundamenta en los resultados de la investigación "Las competencias de los administradores en Colombia a la luz del proyecto Tuning América Latina" realizado por ASCOLFA -GRIICA. El objetivo es determinar en cada una de las regiones como se ha dividido Colombia, de acuerdo con el esquema de ASCOLFA, las competencias genéricas y específicas más importantes y coincidentes para empleadores y graduados de administración. Se aplica un análisis estadístico-descriptivo inferencial para identificar las competencias más importantes para los grupos de interés. Se concluye que Antioquia, Oriente y Suroccidente son las regiones donde hay más coincidencia y que más satisfacen los requerimientos para el empleo, seguidas por Bogotá y el Caribe, el Centro es la que menos cumple. Al concluir, con base en los resultados, se esboza para cada región el perfil que sería requerido para un administrador.

Palabras clave: Competencias genéricas; Competencias específicas; Tuning; empleadores; Egresados de administración.

\section{COMPETENCES IMPORTANCE AND COINCIDENCE FOR MANAGEMENT GRADUATES AND EMPLOYERS: AN APPROACH BY REGIONS IN COLOMBIA}

\begin{abstract}
This document is based on the results of the research "Managers competencies in Colombia in the light of the project Tuning Latin America" carried out by ASCOLFA -GRIICA. The objective is to determine in each of the regions how Colombia has been divided, according to the ASCOLFA plan, the most important and matching generic and specific competences for employers and
\end{abstract}

Artículo de investigación derivado del proyecto: "Las competencias de los administradores en Colombia a la luz del proyecto Tuning América Latina" realizado por ASCOLFA -GRIICA.

* MBA Matemático, Universidad de Antioquia. Especialista en Administración Financiera, Universidad del Norte. Magíster en Administración de Empresas, Universidad de Norte. Docente/Investigadora Escuela de Negocios - Universidad del Norte, BarranquillaColombia. Correo electrónico: lcabeza@uninorte.edu.co

MBA Licenciado en educación, Universidad de San Buenaventura, Medellín. Maestría en Administración, EAFIT Medellín. Profesor Escuela de Negocios, Universidad del Norte, Colombia. Docente/Investigador Escuela de Negocios - Universidad del Norte, Barranquilla-Colombia. Correo electrónico: jcastril@uninorte.edu.co

PhD Economista, Universidad del Rosario. Especialista en Relaciones Internacionales. Maestría en Estudios Internacionales, Universidad de Chile. Doctor en Economía, Universidad de Goettingen, Alemania. Docente/Investigador \& Director Académico de la Escuela de Negocios - Universidad del Norte, Barranquilla-Colombia. Correo electrónico: lombanaj@uninorte.edu.co 
management graduates. A statistical-descriptive inferential analysis is applied to identify the most important competencies for the interest groups. It is concluded that Antioquia, East and Southwest are the regions with more coincidence and satisfy the most the requirements for employment, followed by Bogotá and the Caribbean; the Center is the least compliant. In conclusion and based on the results it outlines for each region the profile that would be required for a manager.

Keywords: Generic competences; Specific competences; Tuning; Employers; Management graduates.

\title{
IMPORTÂNCIA E COINCIDÊNCIA DE COMPETÊNCIAS PARA FORMADOS EM ADMINISTRAÇÃO E EMPREGADORES: UM ENFOQUE POR REGIÕES DA COLÔMBIA
}

\begin{abstract}
Resumo
Este documento se fundamenta nos resultados da pesquisa "As competências dos administradores na Colômbia à luz do projeto Tuning América Latina" realizado por ASCOLFA -GRIICA. O objetivo é determinar em cada uma das regiões da Colômbia, de acordo com o esquema de ASCOLFA, as competências genéricas e específicas mais importantes e coincidentes para empregadores $e$ graduados em administração. Aplica-se uma análise estatístico-descritiva inferencial para identificar as competências mais importantes para os grupos de interesse. Conclui-se que Antioquia, Oriente e Suroccidente são as regiões onde há mais coincidência e que mais satisfazem os requerimentos para o emprego, seguidas por Bogotá e o Caribe, o Centro é a que menos cumpre. Ao concluir, com base nos resultados, esboça-se para a cada região o perfil que seria requerido para um administrador.

Palavras chave: Competências genéricas; Competências específicas; Tuning; Empregadores; Formados em administração.

Cabeza, L., Castrillón, J. \& Lombana, J. (2017) Importancia y coincidencia de competencias para egresados de administración y empleadores: un enfoque por regiones de Colombia. En: Revista de la Facultad de Ciencias Económica: Investigación y Reflexión. rev.fac.cienc.econ, XXV (2), DOI: https://doi.org/10.18359/rfce.1983
\end{abstract}

JEL: A23, M59, J24.

\section{Introducción}

La declaración de Bologna en Europa marca un hito en la evolución de las Instituciones de Educación Superior (IES). Con su proclamación en 1988 (Magna Charta Universitatum) las universidades firmantes buscan promover la libertad investigativa, reglamentaciones del cuerpo profesoral, la formación de calidad de los estudiantes y el intercambio entre universidades. Es precisamente en dicho intercambio donde el proyecto Tuning Educational Structures in Europe
(2000-2010) (González \& Wagenaar, 2006) busca que las universidades afinen sus estructuras para el mejoramiento de la calidad educativa a través de la definición de competencias, la revisión de las equivalencias de créditos académicos y de evaluación. Así en sus primeras fases se definieron las competencias genéricas y específicas de diferentes disciplinas del saber. La administración como una disciplina clave en el estudio de Tuning recibió la respectiva atención (Tuning Academy s.f.) y se trabajó en diferentes partes del mundo, incluyendo Latinoamérica. 
El proyecto Tuning Latinoamérica surge entonces con una motivación similar a la europea para encontrar caminos convergentes en la educación superior y con su propia dinámica ha logrado hacer una revisión de las competencias genéricas y específicas adaptándolas a las regiones estudiadas (Esquetini, 2013). Estos mismos pasos fueron retomados en Colombia por un grupo de investigadores de administración en el marco del convenio llamado ASCOLFA-GRIICA (2010). A partir de la información recolectada en esta investigación han surgido una serie de preguntas sobre las percepciones de los diferentes grupos de interés (estudiantes, docentes, graduados y empleadores) en el marco de la educación superior.

Este documento se centra en determinar en cada una de las regiones en Colombia las competencias genéricas y específicas más importantes para empleadores y graduados de administración, e identificar en ellas las comunes a ambos grupos, así surge el análisis entre lo que solicitan los empleadores como lo más importante para sus empleados y lo que los egresados consideran más importante para su desempeño laboral. En el análisis también se pueden identificar las diferentes percepciones de empleadores y egresados en todas las competencias (ver tablas 5 y 6 ).

Después de esta introducción, en la siguiente sección se hace una revisión sobre los conceptos de competencias, sus tipologías y el estado del arte sobre la investigación en competencias, particularmente en la disciplina administrativa; la tercera parte corresponde a la explicación metodológica descriptiva-inferencial; en la cuarta parte se discute y se hace el análisis de las competencias por regiones; para finalizar con las conclusiones.

\section{Conceptualización sobre las compe- tencias en la disciplina administrativa}

La aplicación académica del concepto de competencia en el ámbito administrativo se remonta a Max Weber (1971) quien define el término cuando dice que aquel que ejerce la función de dominación o autoridad (Behörde) y todos sus funcionarios (burocracia) tienen competencias establecidas (Kompetenz) adecuadas para la labor que desempeñan y ellas se ven respaldadas por pruebas que garanticen su calificación. Sin embargo es con el trabajo de McClelland (1973) quien desde la disciplina de la psicología pone el concepto explícitamente en el ámbito académico, cuando individuos podían calificarse de acuerdo a unas habilidades a las que debían responder. El estudio de las competencias desde este trabajo fue analizado desde diferentes disciplinas pero con The Competent Manager de Boyatzis (1982) vuelve a ser importante en la disciplina administrativa. Para Boyatzis (1982) la competencia es la característica central de un individuo que le permite desempeñarse en una labor o empresa concreta, es bueno anotar que se encuentran gerentes con competencias sobresalientes frente a las que debe tener cualquier directivo.

El debate es amplio por lo que tomando las tipologías que se encuentran en la literatura académica sobre competencias (Hoffmann, 1999 y Ruiz, 2005) se puede dar curso al encuentro de consensos en su definición:

- Conductual, cuando la competencia se refiere a los procesos. Esto se da solamente si se siguen una serie de normas o reglas que ordenen el comportamiento de los individuos (Ducci, 1997; Gallart, 1998 y Mertens, 1997). Para Boyatzis (1982) y Mole, Dawson, Winstanley \& Sherval (1993) el conjunto de normas se da a través de la educación.

- Constructivista, es la potencialidad mental, el desempeño y acciones del individuo las que definen las competencias. Si el contexto es cambiante, el individuo debe tener la capacidad de prever, decidir y adaptarse a la situación (Vigotsky, 1979). La construcción de la competencia es dada por las tareas y lo que demande el contexto (Bunk, 1994; UNESCO, 2011 y Victorino \& Medina, 2008).

- Funcional, es en el resultado donde se verifica la competencia. No importa ni el cómo, ni el qué, siempre y cuando se logre el objetivo (Malpica, 2000 y Koshansky, 1998). Para Gonczi \& Athanasou (1994), atributos, tareas 
y contextos se juntan para conformar las competencias. Por su parte Beneitone, Esqueti, González, Marty, Siufi \& Wagenaar, R. (2007) resumen que las competencias se definen por la conjunción de conocimientos, comprensión, habilidades y capacidades, esta definición abarca los diferentes enfoques encontrados en la literatura y es acogida como la definición en este documento.

Ahora bien, cuando se analizan las percepciones de los grupos de interés de esta investigación, pueden encontrarse diferencias respecto al énfasis tanto de si la competencia se refiere a procesos, contextos o resultados como a la competencia misma que es requerida para cumplir una función en el lugar de trabajo.

Estudios de la correspondencia entre las competencias que solicitan los empleadores y las que ofrecen las instituciones de educación superior con sus egresados son comunes en las diferentes disciplinas y ámbitos de estudio. De hecho es en ese debate de inter-discplinariedad donde surgen las competencias genéricas definidas como aquellas que son necesarias para desempeñar cualquier profesión o labor (González \& Wagenaar, 2003; OECD, 2004).

Por otro lado aparecen las competencias específicas como aquellas que dependen del área concreta de estudio. Una revisión de la literatura entre 2005 y 2015 en la disciplina administrativa y las afines encontró que los estudios que muestran a empleadores y a egresados interesados en las competencias relacionadas con el empleo son más bien pocos. Entre ellos Kavanagh \& Drennan (2008) estudian los atributos de los contadores por una situación coyuntural en las empresas y las normas internacionales que obligaban a una especie de unificación de criterios en términos de los profesionales requeridos por las empresas. Sin embargo en este mismo estudio cuestionan la sobre-especialización de los contadores en aspectos técnicos olvidando competencias transversales y claves como la toma de decisiones.

Por otra parte Wentling (1987), Beach (1982), Cotton (1993), Martensen \& Grønholdt (2009) hacen énfasis en el "graduado global" en las áreas de ad- ministración y economía. En el estudio de estos autores se encuentra que las competencias genéricas como compromiso y motivación para aprender son más importantes que las específicas como conocimientos de gerencia. Además Jackson (2013) concuerda en la percepción de que las competencias genéricas son más importantes que las específicas pero agrega que la transición de la IES a la empresa debe requerir un apoyo colaborativo entre ellas para asegurar el éxito del nuevo empleado.

Otro aspecto es la percepción de empleadores que sí encuentran los trabajadores en el mercado, pero que debido a su limitada capacidad de absorción, las preocupaciones del egresado se centran en los temas de desempleo (Paadi, 2014). Entre los temas de profundización futura en investigación pueden estar los aspectos que cubren la educación en línea, que incluye los MOOC (acrónimo en inglés de Massive Open Online Course), aunque los empleadores todavía no tienen una percepción positiva de las competencias adquiridas a través de estos medios (Fitó-Bertran, Martínez \& Moya, 2014).

Aunque la literatura académica no es pródiga en estudios en que empleadores revisen las competencias de sus futuros empleados, particularmente en administración, coinciden en que las competencias genéricas son las principales a la hora de la selección. Para Willis \& Taylor (1999) se tienen como competencias fundamentales: comunicación, ética, creatividad, espíritu empresarial, tecnología de la información y habilidades computacionales, habilidades interpersonales y habilidades para resolver problemas. Wilton $(2011 ; 2012)$ pondera como las más importantes: comunicación escrita, habilidad para trabajar en equipos y capacidades de investigación. En algunos casos la preocupación por definir las competencias ha pasado al plano de si las instituciones están preparando a sus graduados para la empleabilidad, y si no lo hacen cuáles son las instituciones encargadas para hacerlo. Raybould \& Sheedy (2005), describen cómo organizaciones reclutadoras ofrecen seminarios para fortalecer competencias que requieren los empleadores. Zinser, (2003) va incluso a la creación desde la escuela secundaria, de asignaturas en las que se incluya fortalecimiento de competencias para la emplea- 
bilidad. Por otro lado las revistas especializadas en temas económicos como Forbes, Fortune, The Economist, entre otras que a su vez se nutren de empresas consultoras y cazatalentos como KPMG, Andersen, Addeco, entre otras, realizan de manera periódica estudios con las tendencias laborales en el área disciplinar, coincidiendo en que las empresas se enfrentan a ambientes cada vez más inciertos, los egresados deben estar preparados para afrontarlos y las habilidades más solicitadas cambiarán con las necesidades del mercado (Byrne, 2014; Universia, 2011; The Economist, 2001). Sin embargo aunque se tiene cierta homogeneidad en las competencias deseadas por los empleadores se perciben vacíos en competencias claves como: comunicaciones y pensamiento crítico, que los académicos piensan son importantes (Dishman, 2016).

Para el caso de Colombia el estudio de competencias en el área administrativa es aún más limitado (p.ej: Daza, Charris \& Viloria, 2015; Aguilar-Joyas, 2015; Valero, 2011; Castrillón, Cabeza \& Lombana 2015; Parra-Alviz Ramírez \& Montero 2015) y se derivan precisamente del proyecto ASCOLFAGRIICA (2010) anteriormente reseñado. Adicionalmente en lo que respecta a estudios de percepción de empleadores están reseñados única y exclusivamente en los reportes del Observatorio Laboral para la Educación que por su naturaleza está limitado a datos y noticias de coyuntura (MEN, s.f.). El Ministerio de Educación Nacional sugiere que las competencias que el sistema educativo debe desarrollar en los estudiantes son de tres clases: básicas, ciudadanas y laborales. Para mejorar la calidad de la educación, se requiere un enfoque de una educación más integradora que articule la teoría y la práctica. (MEN, 2010).

\section{Metodología}

Esta es una investigación descriptiva e inferencial, para la cual se toma la muestra de la población conformada por graduados y empleadores de las universidades vinculadas a ASCOLFA, (Asociación Colombiana de Facultades de Administración), se tomó la información de 101 programas de administración adscritos a ASCOLFA clasificados en seis regiones definidas por los seis capítulos en los que
ASCOLFA ha divido el país. Esta división trata de reunir, en un elevado porcentaje, culturas y tipologías similares, los nombres de las regiones también tratan de expresar la ubicación geográfica, ellas son: Antioquia: comprende los departamentos de Antioquia, Caldas y Chocó región ubicada en el Nor-occidente del país. Bogotá: que comprende la ciudad capital con su departamento Cundinamarca y los departamentos de Boyacá, Casanare y el Meta. El Centro comprende los departamentos de Tolima, Huila, Caquetá. El Caribe comprende los departamentos de Atlántico, Bolívar, Cesar, Córdoba, Guajira, Magdalena, Sucre y las Islas de San Andrés y Providencia. El Oriente comprende los departamentos de Norte de Santander y Santander y el Suroccidente comprende los departamentos de Quindío, Risaralda, Valle del Cauca, Cauca y Nariño. Se aplicaron dos cuestionarios a los grupos de estudio empleadores y egresados de las respectivas instituciones, uno para las competencias genéricas y el otro para específicas, donde se indaga la importancia de las competencias identificadas en el proyecto Tuning Latinoamérica, requeridas para el buen desempeño de un administrador.

Se utilizó un instrumento con una escala Likert de 4 , donde 1 es nada importante y 4 es bastante importante, que se construyó y aplicó en el proyecto Tuning Latinoamérica, el cual en su momento fue validado por expertos de los diferentes países participantes; además en ASCOLFA-GRIICA (2010) este instrumento fue revisado y validado por docentes de diferentes capítulos. En este trabajo se realizó la validación interna del instrumento con el Alfa de Crombach que se aplicó a los 20 ítems utilizados en las competencias específicas y a los 27 ítems utilizados en las competencias genéricas que se indagaron con egresados y empleadores. En las competencias Genéricas y Específicas se observa un Alfa de Crombach del $92 \%$ y $91 \%$ respectivamente el cual es el límite inferior de la verdadera fiabilidad del cuestionario. El criterio para definir si este valor del estadístico es aceptable es el siguiente: $\mathrm{Si}$ Alfa de Crombach $>0.9$ es excelente; Alfa de Crombach $>0.8$ es bueno; Alfa de Crombach $>0.7$ es aceptable; Alfa de Crombach $>0.6$ es cuestionable; Alfa de Crombach $>0.5$ es pobre. Luego es excelente la fiabilidad de los dos instrumentos (ver tabla 1). 
Tabla 1. Validación interna de los Instrumentos

\begin{tabular}{|r|r|r|r|r|r|}
\hline \multicolumn{2}{|c|}{$\begin{array}{c}\text { Estadísticas de fiabilidad Competencias Genéricas Gra- } \\
\text { duados y Empleadores }\end{array}$} & \multicolumn{2}{c|}{$\begin{array}{c}\text { Estadísticas de fiabilidad Competencias Específicas - Gra- } \\
\text { duados y Empleadores }\end{array}$} \\
\hline Alfa de Cronbach & $\begin{array}{c}\text { Alfa de Cronbach } \\
\text { basada en elementos } \\
\text { estandarizados }\end{array}$ & N de elementos & Alfa de Cronbach & $\begin{array}{c}\text { Alfa de Cronbach } \\
\text { basada en elementos } \\
\text { estandarizados }\end{array}$ & N de elementos \\
\hline 0,918 & 0,920 & 27 & 0,908 & 0,908 & 20 \\
\hline
\end{tabular}

Fuente: Elaboración propia con el soporte del SPSS 23.

Para determinar el tamaño de la muestra en esta investigación se tomó como base la información de las 101 instituciones vinculadas a ASCOLFA a la fecha del desarrollo de este trabajo, dado que no se logró tener una información completa del número de egresados (de los últimos cinco cohortes) y de empleadores presentados por cada universidad se trabajó bajo el supuesto de población infinita, para esto se utilizó la expresión:

(a) $n_{\alpha}=\frac{P \cdot(1-p) Z_{\alpha}{ }^{2}}{\epsilon^{2}}$

Teóricamente se considera población infinita si $\mathrm{N}>5.000$. Si se tiene población finita (Chow, 1987) y se cumple: $N>n \alpha(n \alpha-1)$ el tamaño muestral calculado con $n_{-} \alpha$ es el correcto, pero si esto no se cumple se debe ajustar el tamaño con: (b) $n=\frac{n_{\propto}}{1+\frac{n_{\alpha}}{N}}$

tamaño muestral para población finita, muy próximo a la expresión;

(c) $n=\frac{N P(1-P) Z_{\alpha}{ }^{2}}{(N-1) \epsilon^{2}+P(1-p) Z_{\alpha}{ }^{2}}$

Para una confianza del 95\% y un margen de error del $5 \%$ y con: $\mathrm{P}=0,5 ; \mathrm{Z}_{-} \alpha=1,96 ; \epsilon=0,05$ y $(1-\alpha)=0,95$ se tienen una muestra de n $\alpha=384$. Como la dificultad era definir el número de egresados del período de estudio se toma la información del ministerio de educación y se encontró la población máxima posible que son los egresados por capítulo en seis años:

Tabla 2. Población de Graduados Programas de Administración 2005-2010 según MEN

\begin{tabular}{|c|c|c|c|c|c|c|c|c|c|c|c|c|c|c|}
\hline \multicolumn{15}{|c|}{ Egresados del 2006 al 2010} \\
\hline Capítulos & 2005 & $\%$ & 2006 & $\%$ & 2007 & $\%$ & 2008 & $\%$ & 2009 & $\%$ & 2010 & $\%$ & Total Graduados & $\%$ \\
\hline Antioquía & 1951 & $12 \%$ & 2133 & $15 \%$ & 2173 & $12 \%$ & 2221 & $12 \%$ & 2508 & $14 \%$ & 3123 & $16 \%$ & 14109 & $15 \%$ \\
\hline El caribe & 1884 & $12 \%$ & 1483 & $11 \%$ & 1936 & $11 \%$ & 2110 & $12 \%$ & 2545 & $14 \%$ & 2946 & $15 \%$ & 12904 & $13 \%$ \\
\hline Bogotá & 6610 & $42 \%$ & 7284 & $52 \%$ & 6949 & $39 \%$ & 7248 & $40 \%$ & 7917 & $44 \%$ & 7415 & $39 \%$ & 43423 & $45 \%$ \\
\hline Centro & 835 & $5 \%$ & 770 & $5 \%$ & 1286 & $7 \%$ & 944 & $5 \%$ & 1194 & $7 \%$ & 1219 & $6 \%$ & 6248 & $6 \%$ \\
\hline Sur occidente & 1738 & $11 \%$ & 1738 & $12 \%$ & 2320 & $13 \%$ & 2540 & $14 \%$ & 3025 & $17 \%$ & 3374 & $18 \%$ & 14735 & $15 \%$ \\
\hline El Oriente & 683 & $4 \%$ & 649 & $5 \%$ & 1122 & $6 \%$ & 930 & $5 \%$ & 823 & $5 \%$ & 1122 & $6 \%$ & 5329 & $6 \%$ \\
\hline & 15706 & & 14057 & & 17793 & & 18001 & & 18012 & & 19199 & & 96748 & \\
\hline
\end{tabular}

Fuente: Elaboración propia con datos del MEN. 
Como: $96.748<384(384-1)=147.072$, se debe aplicar el factor de población finita:

$$
n>\frac{384}{1+\frac{384}{96.748}}=382
$$

Para este estudio se trabajó con muestras de graduados de 952 y 999 para las competencias genéricas y especificas respectivamente (ver tabla 3).
Como el número de graduados de los 101 programas vinculados a ASCOLFA es mucho menor al total del Ministerio de Educación Nacional de Colombia-MEN (ver tabla 2) dado que no todos los programas están en ASCOFA, podemos concluir que la muestra es suficientemente grande y representativa; supera el tamaño mínimo de cada capítulo correspondiente al tamaño muestral recomendado de 382 graduados. Se utilizó el porcentaje de graduados totales suministrado por el ministerio de educación.

Tabla 3. Muestra - Competencias Genéricas y Específicas Graduados

\begin{tabular}{|l|l|l|l|l|l|}
\hline \multirow{2}{*}{ Capítulos } & \multicolumn{5}{|c|}{ Graduados } \\
\cline { 2 - 6 } & Genéricas & $\%$ & Especificas & $\%$ & Tamaño Mínimo Con 382 \\
\hline Antioquía & 78 & $8 \%$ & 78 & $8 \%$ & 56 \\
\hline Bogotá & 234 & $25 \%$ & 255 & $26 \%$ & 171 \\
\hline Caribe & 370 & $39 \%$ & 406 & $41 \%$ & 51 \\
\hline Centro & 57 & $6 \%$ & 63 & $6 \%$ & 25 \\
\hline Oriente & 53 & $6 \%$ & 52 & $5 \%$ & 21 \\
\hline Suroccidente & 160 & $17 \%$ & 145 & $15 \%$ & 58 \\
\hline & 952 & & 999 & & \\
\hline
\end{tabular}

Fuente: Construcción autores.

Para el caso de empleadores fue mucho más difícil definir el tamaño poblacional, luego se partió del supuesto de población infinita: Para $\mathrm{P}=0,08 ; \mathrm{Z} \alpha=$ 1,$96 ; \epsilon=0,05$ y $(1-\alpha)=0,95$ se tienen una muestra de $n \alpha=150$. Aplicando la condición para definir si esta muestra es correcta se tiene que: $\mathrm{N}>150(150-$ $1)=22.350$; teniendo en cuenta lo reportado por el observatorio laboral, donde hacen referencia a una población de 40.230, esto para 12 ciudades: Medellin, Barranquilla, Cartagena, Manizales, Montería, Villavicencio, Pasto, Cúcuta, Armenia, Bucaramanga, Ibagué, Cali y Bogotá (MEN, 2008-2013), podemos afirmar con base en esto que el tamaño muestral mínimo para nosotros es de 150 empleadores y está bien el supuesto de población infinita. Se trabajó con una muestra de 219 y 238 empleadores para genéricas y especificas respectivamente (ver tabla 4), luego es representativa la muestra tomada.
Tabla 4. Muestra - Competencias Genéricas y Específicas Empleadores

\begin{tabular}{|l|l|c|l|c|}
\hline & \multicolumn{4}{|c|}{ Empleadores } \\
\hline \multicolumn{1}{|c|}{ Capitulo } & Genéricas & $\%$ & Especificas & $\%$ \\
\hline Antioquía & 36 & $16 \%$ & 36 & $15 \%$ \\
\hline Bogotá & 14 & $6 \%$ & 34 & $14 \%$ \\
\hline El caribe & 72 & $33 \%$ & 75 & $32 \%$ \\
\hline Centro & 32 & $15 \%$ & 33 & $14 \%$ \\
\hline El Oriente & 23 & $11 \%$ & 24 & $10 \%$ \\
\hline Sur occidente & 42 & $19 \%$ & 36 & $15 \%$ \\
\hline & 219 & & 238 & \\
\hline
\end{tabular}

Fuente: Elaboración propia. 
Con la información de las muestras se procedió a realizar una inferencia para la diferencia de los promedios de importancia de cada competencia en los dos grupos (Empleadores y Graduados) y tipo de competencia (Genéricas y Especificas). Además, con el apoyo de la estimación puntual, se identificaron las cinco competencias que tienen el mayor promedio de importancia para cada grupo y en cada tipo de competencia. Se utilizó una prueba de hipótesis a un nivel de significancia del 5\%, lo cual permitió identificar las competencias que presentan diferente media.

Para realizar lo anterior se procedió a verificar los supuestos requeridos para la docimasia de diferencia de medias: (1) Probar el comportamiento de las varianzas de las poblaciones de graduados y de empleadores, para 27 competencias genéricas y 20 específicas. Se aplicó una prueba de hipótesis bilateral para la igualdad de varianzas poblacionales esto es: $\mathrm{H}_{0}:$ Graduados $={ }_{\text {Empleadores }} \mathrm{y} \mathrm{H}_{1}:_{\text {Graduados }} \neq$ Empleadores , dado que en este trabajo se utilizó el SPSS 23, el cual maneja en la prueba de igualdad de medias para poblaciones independientes la prueba de Levene para la docimasia de igualdad de varianzas poblacionales, dado que este estadístico es menos dependiente del supuesto de normalidad; se identificaron las competencias que presentaron un $\mathrm{P}$-valor, menor al 5\%, (estas competencias se presentan en la tabla $5 \mathrm{y}$ tabla 6 identificadas con un diamante (»). (2) El supuesto de normalidad de la distribución muestral de diferencia de medias se verifica por el teorema del límite central (Chow, 1987), ya que el tamaño de las muestras tomadas son mayores a 30 datos.

Habiendo comprobado los supuestos se procedió a realizar la docimasia: ${ }_{\mathrm{H} 0}$ : $\mu$ Graduados $=\mu$ Empleadores $\mathrm{y} \mathrm{H}_{1}$ : $\mu_{\text {Graduados }} \neq \mu_{\text {Empleadores: }}$ para las competencias con un P-valor, menor al $5 \%$ es significativa la diferencia de medias poblacionales, (en las tablas 5 y 6 se iden- tifican con trébol \&) y con el apoyo del intervalo de confianza se identificaron las competencias que presentaban una mayor importancia para graduados o empleadores. El promedio de importancia puntual asociado en cada grupo a cada una de las competencias (genéricas y específicas) por región geográfica se observa en las tablas 5 y 6 respectivamente. Las identificadas con corazones $(\boldsymbol{})$ son las cinco competencias con mayor importancia puntual por grupo. Dichas tablas resumen los resultados de las pruebas estadísticas que se han descrito para las competencias genéricas y específicas en cada región geográfica seleccionada: Antioquia, Bogotá, Caribe, Centro, Oriente y Suroccidente.

\section{Análisis y Resultados}

En la primera parte de esta sección se presentan los resultados para las competencias genéricas, posteriormente se hace lo mismo para las específicas.

En la tabla 5 se presentan las 27 competencias genéricas. Aquellas señaladas con un rombo ( $\bullet$ son las que presentan diferencias significativas entre las varianzas poblacionales para empleadores y graduados; las que no están identificadas con el rombo presentan varianzas poblacionales iguales, de acuerdo a la prueba de hipótesis de igualdad de varianzas poblacionales según Leven. El suroccidente con $59,26 \%$ y el centro con $44,44 \%$ presentan más competencias con varianzas poblacionales diferentes. En Bogotá y el Caribe un 18,52\% de las competencias presentan varianzas poblacionales diferentes. El Oriente con 37,04\% y Antioquia con $33,33 \%$ presentan varianzas poblacionales diferentes.

Las identificadas con trébol (\$) son las que presentan diferente media poblacional de acuerdo a la estimación puntual. 
IMPORTANCIA Y COINCIDENCIA DE COMPETENCIAS PARA EGRESADOS DE ADMINISTRACIÓN Y EMPLEADORES:

UN ENFOQUE POR REGIONES DE COLOMBIA

\begin{tabular}{|c|c|c|c|c|c|c|c|c|c|c|c|c|c|c|}
\hline \multirow{2}{*}{ 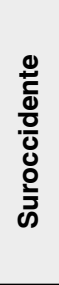 } & 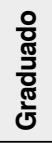 & $\begin{array}{l}\stackrel{\leftrightarrow}{0} \\
\stackrel{m}{0}\end{array}$ & $\begin{array}{l}\stackrel{b}{\circ} \\
\stackrel{m}{m}\end{array}$ & $\begin{array}{l}\mathbb{U} \\
\tilde{m}\end{array}$ & 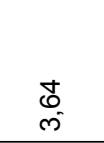 & 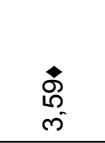 & $\begin{array}{l}\& \\
\stackrel{\infty}{0}\end{array}$ & $\stackrel{\text { F }}{\infty}$ & $\begin{array}{l}: \\
\mathbb{8} \\
\text { m. }\end{array}$ & $\stackrel{?}{\stackrel{9}{m}}$ & $\begin{array}{l}\bar{\sigma} \\
\tilde{m}^{-}\end{array}$ & $\begin{array}{l}\stackrel{\circ}{\circ} \\
\text { م }\end{array}$ & $\begin{array}{l}\text { Lे } \\
\text { m. }\end{array}$ & $\begin{array}{l}1 \\
0 \\
0 \\
0 \\
\infty \\
0\end{array}$ \\
\hline & $\begin{array}{l}\text { 흥 } \\
\text { Ð } \\
\text { 음 } \\
\text { ㄴ }\end{array}$ & $\begin{array}{l}\stackrel{0}{0} \\
m \\
m\end{array}$ & $\begin{array}{l}\bar{\kappa} \\
m\end{array}$ & $\begin{array}{l}\vec{j} \\
\hat{m}\end{array}$ & $\begin{array}{l}\hat{0} \\
\tilde{m}\end{array}$ & $\begin{array}{l}\stackrel{+}{N} \\
\dot{m}\end{array}$ & $\begin{array}{l}8 \\
m\end{array}$ & $\begin{array}{l}\widetilde{N} \\
\text { ల) }\end{array}$ & $\begin{array}{l}\text { 苫 } \\
\text { ल }\end{array}$ & ర్ & $\begin{array}{l}\Xi \\
\infty\end{array}$ & $\begin{array}{l}\vec{N} \\
\dot{m}\end{array}$ & 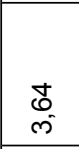 & $\begin{array}{l}+ \\
0 \\
0 \\
\infty \\
m\end{array}$ \\
\hline \multirow{2}{*}{ 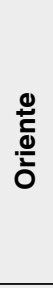 } & 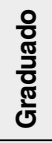 & $\begin{array}{l}\text { है } \\
\stackrel{0}{0}\end{array}$ & $\begin{array}{l}\vec{\infty} \\
\dot{\infty} \\
\end{array}$ & $\begin{array}{l}\stackrel{N}{N} \\
m\end{array}$ & $\frac{+}{+}$ & $\begin{array}{l}\mathbb{N} \\
m\end{array}$ & $\begin{array}{l}\stackrel{L}{2} \\
m^{-}\end{array}$ & 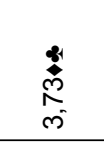 & $\begin{array}{l}\stackrel{2}{\circ} \\
m\end{array}$ & 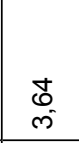 & $\begin{array}{l}\dot{0} \\
\infty \\
\tilde{m}\end{array}$ & $\begin{array}{c}2 \\
\hat{n} \\
m\end{array}$ & 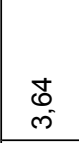 & $\begin{array}{c}R \\
m \\
m\end{array}$ \\
\hline & $\begin{array}{l}\text { 흠 } \\
\text { ळ } \\
\text { 음 } \\
\text { జ }\end{array}$ & $\begin{array}{l}\text { के } \\
\text { లె }\end{array}$ & $\begin{array}{l}\stackrel{\leftrightarrow}{0} \\
\stackrel{m}{0}\end{array}$ & $\begin{array}{l}\hat{\omega} \\
m\end{array}$ & $\begin{array}{l}\text { 今े } \\
\text { ले } \\
\end{array}$ & $\begin{array}{l}\bar{\sigma} \\
\text { - }\end{array}$ & 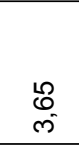 & \begin{tabular}{c}
$\stackrel{+}{*}$ \\
\multirow{2}{*}{}
\end{tabular} & $\begin{array}{l}\text { గ్ } \\
\text { ల) }\end{array}$ & $\begin{array}{l}\text { న్ } \\
\text { లn }\end{array}$ & $\begin{array}{l}\text { हे } \\
\text { m. }\end{array}$ & 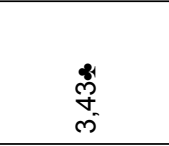 & $\begin{array}{l}\text { న్ } \\
\text { లె }\end{array}$ & $\begin{array}{c}\overline{6} \\
\dot{m}\end{array}$ \\
\hline \multirow{2}{*}{ 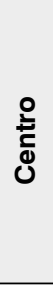 } & 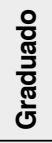 & $\underset{\hat{N}}{\stackrel{\dot{N}}{n}}$ & $\begin{array}{l}: \\
\stackrel{5}{N} \\
m \\
m\end{array}$ & $\begin{array}{l}\hat{\omega} \\
\tilde{m}\end{array}$ & $\begin{array}{l}\stackrel{0}{0} \\
\stackrel{m}{0}\end{array}$ & $\begin{array}{l}R \\
m\end{array}$ & $\begin{array}{l}\vdots \\
\infty \\
\infty \\
m\end{array}$ & $\begin{array}{l}+ \\
\stackrel{1}{*} \\
\stackrel{m}{0}\end{array}$ & 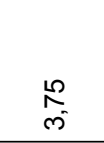 & $\begin{array}{l}8 \\
0 \\
\infty\end{array}$ & $\begin{array}{l}\stackrel{\infty}{\stackrel{m}{m}} \\
\text { - }\end{array}$ & $\begin{array}{l}\stackrel{\vec{N}}{m} \\
\text { - }\end{array}$ & $\begin{array}{l}\hat{6} \\
\dot{m}\end{array}$ & $\begin{array}{l}\infty \\
\vdots \\
\infty\end{array}$ \\
\hline & $\begin{array}{l}\text { 흠 } \\
\text { ळ } \\
\text { 을 } \\
\text { ㅍ }\end{array}$ & 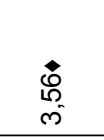 & $\begin{array}{l}\ddot{8} \\
\ddot{\infty}\end{array}$ & $\begin{array}{l}8 \\
\text { m }\end{array}$ & 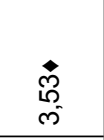 & $\begin{array}{l}\text { B) } \\
\text { m) }\end{array}$ & 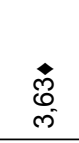 & 今े & 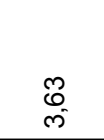 & $\begin{array}{l}\infty \\
\stackrel{\infty}{\infty} \\
\infty\end{array}$ & $\begin{array}{l}\text { ஜ } \\
\text { ల. }\end{array}$ & $\begin{array}{l}\stackrel{0}{0} \\
\text { m }\end{array}$ & $\begin{array}{l}\infty \\
\stackrel{\infty}{\infty} \\
\stackrel{n}{n}\end{array}$ & $\begin{array}{l}\stackrel{0}{\infty} \\
\stackrel{\infty}{\infty}\end{array}$ \\
\hline \multirow{2}{*}{ 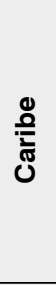 } & 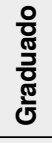 & $\begin{array}{l}5 \\
0 \\
\infty\end{array}$ & ¿ & $\begin{array}{l}\infty \\
\infty \\
\infty \\
\infty \\
\infty \\
\infty\end{array}$ & $\begin{array}{l}\dot{\tilde{U}} \\
\dot{m}\end{array}$ & 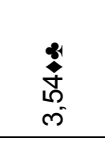 & 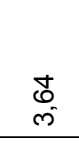 & $\begin{array}{l}0 \\
m \\
m\end{array}$ & $\begin{array}{l}0 \\
\infty \\
\infty\end{array}$ & $\begin{array}{l}0 \\
m \\
m\end{array}$ & $\begin{array}{l}3 \\
\text { m }\end{array}$ & $\begin{array}{l}0 \\
\stackrel{m}{m} \\
\end{array}$ & $\begin{array}{l}\hat{m} \\
\text { f }\end{array}$ & $\begin{array}{l}\infty \\
\stackrel{\infty}{\infty} \\
m\end{array}$ \\
\hline & $\begin{array}{l}\text { 흥 } \\
\text { ळ } \\
\text { 을 } \\
\text { w }\end{array}$ & 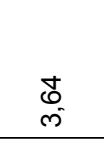 & $\begin{array}{l}2 \\
0 \\
0 \\
m\end{array}$ & 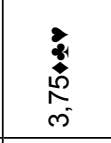 & $\begin{array}{l}\dot{N} \\
m\end{array}$ & $\begin{array}{l}4 \\
0 \\
0 \\
\infty \\
\infty\end{array}$ & 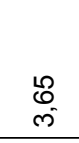 & $\begin{array}{l}\forall \\
m\end{array}$ & $\begin{array}{l}\infty \\
\infty \\
\sim^{\circ}\end{array}$ & $\begin{array}{l}0 \\
\infty \\
\infty\end{array}$ & $\begin{array}{l}\widetilde{\delta} \\
\text { m }\end{array}$ & $\begin{array}{l}\infty \\
\infty \\
\infty \\
m\end{array}$ & $\begin{array}{l}\hat{b} \\
m^{2}\end{array}$ & $\begin{array}{l}8 \\
\text { m. }\end{array}$ \\
\hline \multirow{2}{*}{ 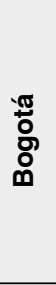 } & $\begin{array}{l}\text { 융 } \\
\frac{\pi}{0} \\
\frac{\pi}{0} \\
\text { U. }\end{array}$ & 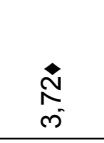 & $\stackrel{M}{\stackrel{M}{m}}$ & $\begin{array}{l}\qquad 0 \\
\text { ల }\end{array}$ & $\begin{array}{l}\mathscr{0} \\
\text { m }\end{array}$ & $\begin{array}{l}\text { ¿ैं } \\
\text { m }\end{array}$ & 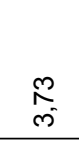 & ॄే & $\begin{array}{l}\hat{0} \\
\dot{m}\end{array}$ & $\begin{array}{l}\stackrel{L}{L} \\
\text { m. }\end{array}$ & 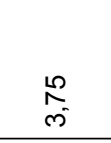 & $\begin{array}{l}\stackrel{8}{6} \\
\text { n) }\end{array}$ & $\begin{array}{l}8 \\
0 \\
0\end{array}$ & $\begin{array}{c}\hat{m} \\
\dot{m}\end{array}$ \\
\hline & $\begin{array}{l}\text { 흠 } \\
\text { ळ } \\
\text { 음 } \\
\text { ㅍ }\end{array}$ & $\begin{array}{l}: \\
0 \\
\infty \\
\infty \\
m\end{array}$ & $\begin{array}{l}\bar{N} \\
m\end{array}$ & $\begin{array}{l}\Xi \\
\text { ల }\end{array}$ & $\begin{array}{l}8 \\
\text { m } \\
\end{array}$ & 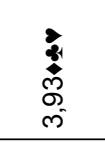 & $\begin{array}{l}8 \\
\infty \\
\infty \\
\end{array}$ & $\begin{array}{l}\hat{\omega} \\
0 \\
0\end{array}$ & 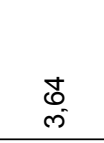 & $\begin{array}{l}n \\
\text { nj }\end{array}$ & $\begin{array}{l}\infty \\
\infty \\
\text { m. }\end{array}$ & $\begin{array}{c}\bar{n} \\
m\end{array}$ & \begin{tabular}{l}
\multirow{\sigma}{*}{} \\
ल
\end{tabular} & 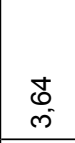 \\
\hline \multirow{2}{*}{ 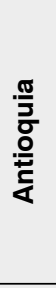 } & 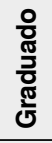 & $\begin{array}{l}\stackrel{0}{0} \\
\text { m }\end{array}$ & $\begin{array}{l} \\
\vdots \\
\infty \\
\infty\end{array}$ & $\begin{array}{l}\stackrel{8}{6} \\
\text { m }\end{array}$ & 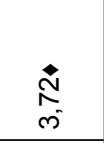 & $\begin{array}{l}1 \\
0 \\
0 \\
0 \\
0\end{array}$ & $\begin{array}{l}\overline{0} \\
\text { N. }\end{array}$ & $\begin{array}{l}\stackrel{\circ}{m} \\
\text { ल }\end{array}$ & $\begin{array}{l}\text { م) } \\
\text { n) }\end{array}$ & 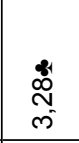 & $\begin{array}{l}\hat{0} \\
\tilde{\omega}\end{array}$ & $\begin{array}{l}0 \\
\stackrel{\infty}{\infty} \\
\infty\end{array}$ & $\stackrel{\text { fo }}{\stackrel{m}{m}}$ & 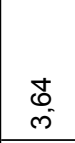 \\
\hline & $\begin{array}{l}\text { 윯 } \\
\text { ळ } \\
\text { ్ㅡㄹ } \\
\text { w }\end{array}$ & $\begin{array}{l}\stackrel{+}{N} \\
\dot{m}\end{array}$ & $\begin{array}{l}\dot{0} \\
\dot{m}\end{array}$ & $\underset{\hat{N}}{\stackrel{\dot{N}}{m}}$ & 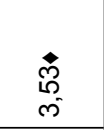 & 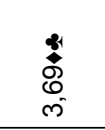 & $\begin{array}{l}8 \\
0\end{array}$ & $\underset{m}{\stackrel{m}{m}}$ & $\begin{array}{l}0 \\
\stackrel{\infty}{\infty} \\
m\end{array}$ & $\begin{array}{l}\text { s. } \\
\text { مी } \\
\text { ch }\end{array}$ & $\begin{array}{l}\hat{0} \\
\tilde{m}\end{array}$ & $\begin{array}{l}\hat{0} \\
\hat{m}\end{array}$ & $\begin{array}{l}0 \\
\stackrel{\infty}{\infty} \\
\tilde{\infty}\end{array}$ & $\begin{array}{l}\stackrel{8}{0} \\
\text { i }\end{array}$ \\
\hline & 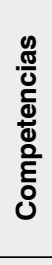 & 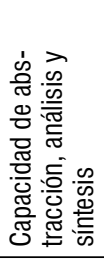 & 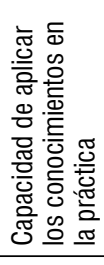 & 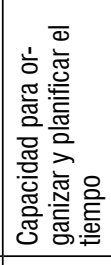 & 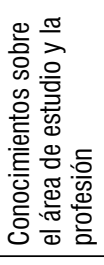 & 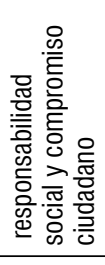 & 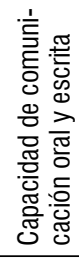 & 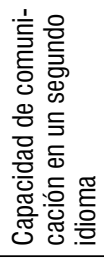 & 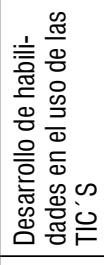 & 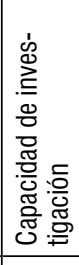 & 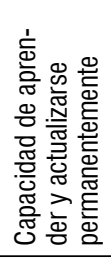 & 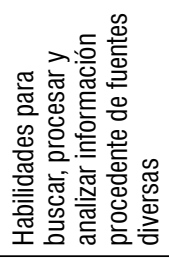 & 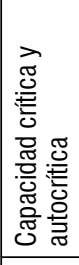 & 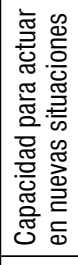 \\
\hline & * & - & $\sim$ & $m$ & $\nabla$ & ما & 0 & $\Lambda$ & $\infty$ & $\sigma$ & 으 & $\mp$ & $\simeq$ & $\stackrel{m}{\stackrel{2}{2}}$ \\
\hline
\end{tabular}




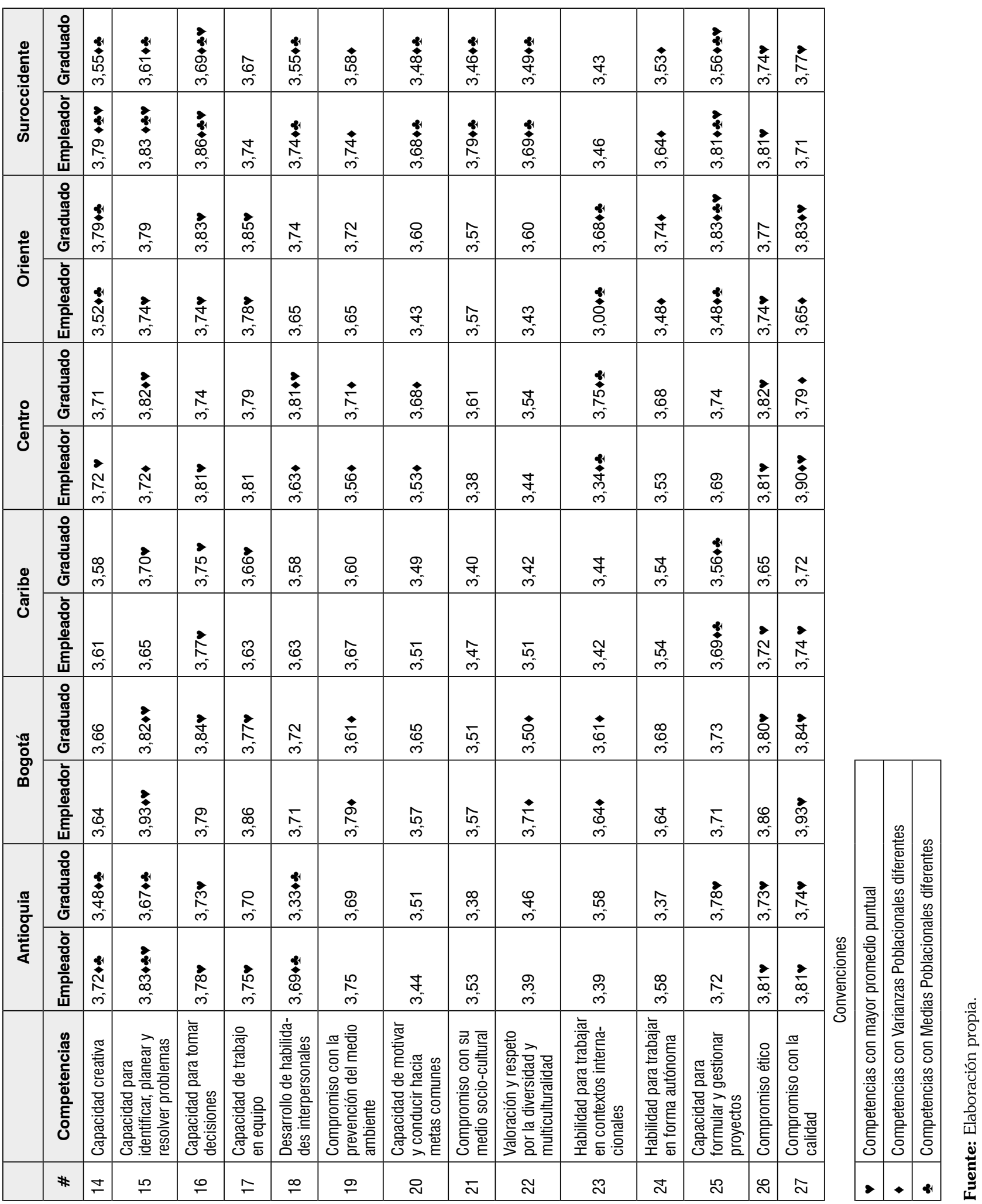


En Antioquia las competencias genéricas más importantes para empleadores y graduados coinciden en 3 de 5 (16, 26 y 27). La importancia promedio para las competencias, para en empleadores oscila entre $[3,31 ; 3,83]$ y en graduados $[3,28 ; 3,81]$. Los empleadores dan mayor promedio. En las competencias $5,9,14,15$ y 18 ; se puede afirmar que es significativa la diferencia en medias poblacionales.

En Bogotá coinciden en 2 de 5 (15 y 27). La importancia para empleadores y graduados oscila entre $[3,50 ; 3,93]$ y $[3,50 ; 3,84]$ respectivamente. De todas las competencias en la (5), es significativa la diferencia entre los promedios poblacionales.

En el Caribe coinciden en 3 de 5 (2, 16 y 27). La importancia promedio oscila dentro de $[3,42 ; 3,77]$ y $[3,36 ; 3,74]$ para empleadores y graduados respectivamente. De estas competencias solo para la 3,5 y 25 se puede afirmar que el promedio de importancia es diferente.

En el Centro coinciden en la 26. La importancia promedio de las competencias oscila dentro de $[3,03 ; 3,90]$ y $[3,54 ; 3,82]$ para empleadores y graduados respectivamente, los empleadores dan mayor ponderación puntual. La diferencia en la percepción de importancia para los dos grupos en las competencias 7 y 23 es significativa.

En el Oriente comparten la 10, 16 y 17, con una coincidencia de 3 sobre 5 para graduados y empleadores. La importancia promedio de las competencias oscila dentro de $[2,74 ; 3,78]$ y $[3,57 ; 3,86]$ para empleadores y graduados respectivamente, los graduados dan mayor ponderación puntual. En el Oriente las competencias con diferente percepción son: $1,4,7,11,14,23$ y 25.

En el Suroccidente comparten 2 de 5 (16 y 26). La importancia promedio de las competencias oscila dentro de $[3,32 ; 3,86]$ y $[3,42 ; 3,77]$ para empleadores y graduados respectivamente. En el Surocci- dente las competencias con medias diferentes son: $13,14,15,16,18,20,21,22$ y 25.

Comparando las seis regiones geográficas se encontró que las competencias genéricas en las poblaciones de empleadores y graduados que muestran diferente varianza poblacional en la opinión de los dos grupos son: 1, 2, 4, 5 y 15 en cuatro de las regiones es significativa dicha diferencia, es decir en el $66.67 \%$ de las regiones. Para el $50 \%$ de las regiones en las competencias: 3, 7, 14, 18, 19, 23 y 25, las varianzas poblacionales son diferentes.

Mirando la importancia asignada, las competencias genéricas en los dos grupos y en las seis regiones que presentan diferente promedio son: 5,14 y 25 , en tres de las seis regiones son diferentes, es decir en el $50 \%$ de las regiones.

Las competencias genéricas con la mayor importancia en las seis regiones para empleadores y graduados son: capacidad para identificar planear y resolver problemas (15); capacidad de tomar decisiones (16) y compromiso con la calidad (27), estas competencias se presentan en las seis regiones, por lo menos en uno de los dos grupos. Compromiso ético (26) es recurrente en cinco de las regiones. La capacidad de aplicar el conocimiento en la práctica (2) y la capacidad de trabajo en equipo (17) se incluye en cuatro de las regiones por lo menos en uno de los dos grupos. La capacidad para formular y gestionar proyectos (25) se encuentra en tres regiones por lo menos en uno de los grupos.

A continuación, para el análisis de las 20 competencias específicas presentadas en la tabla 6 , se sigue la misma metodología y convenciones establecidas en las competencias genéricas. En el Suroccidente y Oriente se presenta la mayor cantidad de competencias específicas con diferente varianza poblacional ( $\diamond$. Para el Suroccidente es el $90 \%$ y para, el oriente es el $55 \%$, le sigue el Caribe con un $30 \%$, Antioquia 20\%, Centro 20\% y Bogotá 5\%. 


\begin{tabular}{|c|c|c|c|c|c|c|c|c|c|c|c|}
\hline \multirow{2}{*}{ 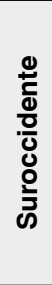 } & $\begin{array}{l}\circ \\
\text { 유 } \\
\frac{\pi}{0} \\
\frac{\pi}{0} \\
\text { U. }\end{array}$ & $\begin{array}{l}\vdots \\
\vdots \\
\vdots \\
\infty\end{array}$ & $\frac{\dot{s}}{\dot{*}}$ & 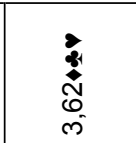 & $\stackrel{\overrightarrow{\hat{n}}}{\mathrm{n}}$ & \& & 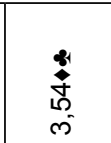 & 离 & 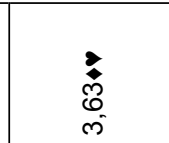 & 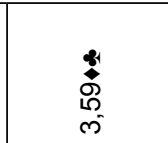 & 惢 \\
\hline & $\begin{array}{l}\text { 흥 } \\
\text { ฐ } \\
\text { 음 } \\
\text { w }\end{array}$ & 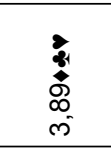 & $\begin{array}{l} \\
\vdots \\
0 \\
0 \\
\infty\end{array}$ & $\begin{array}{l} \\
\$ \\
0 \\
\infty \\
\infty \\
\infty\end{array}$ & $\stackrel{\stackrel{N}{N}}{m}$ & $\begin{array}{l} \\
\infty \\
\infty \\
\infty \\
\infty\end{array}$ & $\begin{array}{l}\$ \\
\stackrel{3}{3} \\
\infty \\
\text { ल) }\end{array}$ & 节 & $\stackrel{\stackrel{\circ}{\circ}}{\tilde{n}^{-}}$ & $\begin{array}{l}\infty \\
\infty \\
\infty \\
\infty \\
\infty\end{array}$ & $\begin{array}{l}\$ \\
\infty \\
\infty \\
\infty\end{array}$ \\
\hline \multirow{2}{*}{ 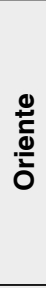 } & 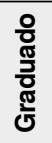 & 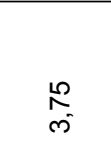 & $\begin{array}{l}\vdots \\
\infty \\
\infty \\
m\end{array}$ & $\begin{array}{l}\overrightarrow{0} \\
\dot{0} \\
\dot{m}\end{array}$ & 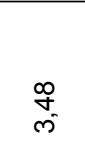 & $\begin{array}{l}\overrightarrow{0} \\
\dot{0} \\
\dot{m}\end{array}$ & $\begin{array}{l}8 \\
\text { r. }\end{array}$ & 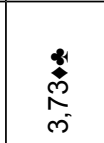 & $\stackrel{3}{m^{2}}$ & $\begin{array}{l} \\
\vdots \\
0 \\
\infty \\
\infty \\
\infty\end{array}$ & 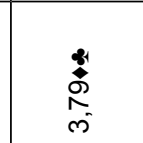 \\
\hline & 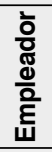 & ले & 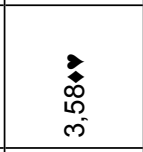 & $\stackrel{\vec{y}}{\stackrel{\sim}{r}}$ & $\begin{array}{l}\stackrel{\infty}{m} \\
m \\
m\end{array}$ & $\begin{array}{l}\dot{0} \\
\dot{m} \\
m\end{array}$ & $\begin{array}{l}\text { m } \\
\text { m }\end{array}$ & $\begin{array}{l}\text { N } \\
\stackrel{N}{N} \\
\text { m. }\end{array}$ & N & $\begin{array}{l}\$ \\
\infty \\
m \\
m \\
m\end{array}$ & $\underset{\stackrel{*}{*}}{\stackrel{\$}{*}}$ \\
\hline \multirow{2}{*}{ 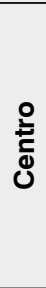 } & 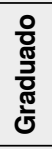 & $\begin{array}{l}: \\
\stackrel{0}{\circ} \\
m \\
\end{array}$ & $\underset{⿱}{\stackrel{0}{n}}$ & $\begin{array}{l}0 \\
0 \\
0\end{array}$ & $\begin{array}{l}0 \\
\stackrel{1}{1} \\
\text { ro }\end{array}$ & 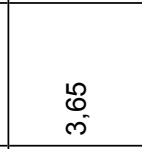 & $\begin{array}{l}\text { m } \\
\text { m. }\end{array}$ & $\begin{array}{l}\stackrel{\text { g }}{+} \\
\text { res }\end{array}$ & $\begin{array}{l}\hat{0} \\
\dot{m}\end{array}$ & $\stackrel{\vec{m}}{\stackrel{m}{m}}$ & 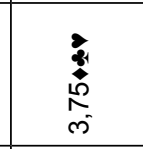 \\
\hline & $\begin{array}{l}\text { 흥 } \\
\text { ฐ } \\
\text { 음 } \\
\text { ㄴ }\end{array}$ & $\begin{array}{l}\infty \\
\infty \\
\infty \\
\infty \\
m\end{array}$ & 芯 & $\begin{array}{l}\stackrel{L}{L} \\
\text { లn }\end{array}$ & 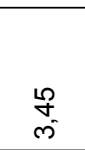 & $\begin{array}{l}R \\
0 \\
0\end{array}$ & 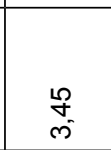 & 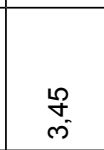 & 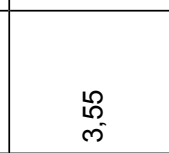 & 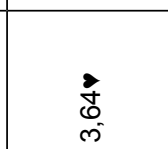 & 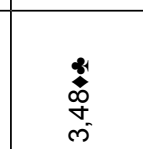 \\
\hline \multirow{2}{*}{ 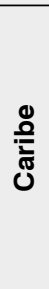 } & 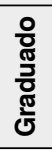 & 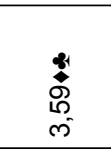 & $\begin{array}{c}\overline{6} \\
\bar{\infty}\end{array}$ & 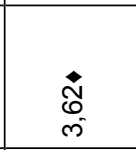 & $\begin{array}{l}\text { o } \\
\text { c }\end{array}$ & $\begin{array}{l}\hat{b} \\
m\end{array}$ & $\begin{array}{l}\mathbb{J} \\
\text { m }\end{array}$ & $\begin{array}{l}\text { o } \\
\text { m }\end{array}$ & $\begin{array}{l}\text { 员 } \\
\text { 足 }\end{array}$ & $\begin{array}{l}2 \\
\hat{6} \\
\dot{n}\end{array}$ & $\stackrel{\dot{0}}{\dot{m}}$ \\
\hline & 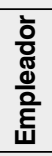 & 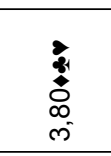 & $\begin{array}{l}\infty \\
@ \\
\overbrace{}^{-}\end{array}$ & $\begin{array}{l}\text { : } \\
\stackrel{5}{\circ} \\
m^{2}\end{array}$ & $\begin{array}{l}8 \\
\text { m. }\end{array}$ & $\begin{array}{l}\hat{6} \\
\tilde{m}\end{array}$ & $\begin{array}{l}\infty \\
\stackrel{\infty}{\infty} \\
\sim\end{array}$ & o & 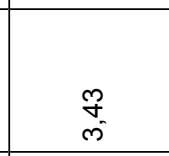 & $\begin{array}{l}\overline{6} \\
\infty\end{array}$ & 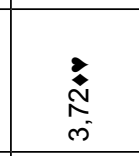 \\
\hline \multirow{2}{*}{ 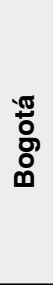 } & 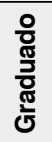 & 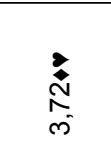 & $\begin{array}{l}R \\
m\end{array}$ & $\begin{array}{l}\text { ठ } \\
\text { m. }\end{array}$ & $\underset{m}{\stackrel{m}{\infty}}$ & $\frac{81}{6}$ & 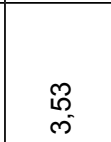 & 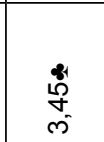 & $\begin{array}{l}\tilde{E} \\
\text { en }\end{array}$ & $\frac{\stackrel{b}{h}}{\stackrel{n}{m}}$ & $\begin{array}{l}\infty \\
\stackrel{\infty}{\infty} \\
\Gamma^{\prime}\end{array}$ \\
\hline & 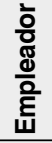 & $\begin{array}{l}\text { : } \\
\infty \\
\infty \\
\infty \\
m\end{array}$ & $\stackrel{\vec{N}}{\stackrel{2}{m}}$ & 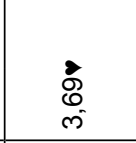 & ले & $\underset{N}{\stackrel{2}{N}}$ & $\begin{array}{l}\text { o+ } \\
\text { m }\end{array}$ & $\frac{+}{\dot{r}}$ & $\begin{array}{l}8 \\
\text { m }\end{array}$ & $\begin{array}{l}8 \\
\text { ల }\end{array}$ & $\begin{array}{l}\hat{\omega} \\
\infty\end{array}$ \\
\hline \multirow{2}{*}{ 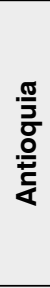 } & 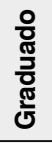 & $\begin{array}{l}8 \\
0 \\
0 \\
\infty\end{array}$ & $\begin{array}{l}\stackrel{L}{L} \\
\text { N }\end{array}$ & 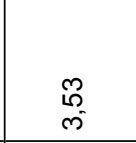 & $\begin{array}{l}\hat{m} \\
\text { n. }\end{array}$ & $\begin{array}{l}\widetilde{\sigma} \\
\text { r }\end{array}$ & $\begin{array}{l}\stackrel{\circ}{\infty} \\
\text { లn }\end{array}$ & $\begin{array}{c}\text { m. } \\
\text { n}\end{array}$ & हु & $\begin{array}{l}\overrightarrow{\mathrm{O}} \\
\text { ल) }\end{array}$ & $\underset{\text { m }}{\stackrel{J}{+}}$ \\
\hline & $\begin{array}{l} \\
\text { 흉 } \\
\mathbb{\Xi} \\
\text { 을 } \\
\text { w }\end{array}$ & $\underset{\substack{R \\
m}}{\stackrel{2}{n}}$ & $\begin{array}{l}\text { B } \\
\text { 心 }\end{array}$ & $\begin{array}{l}\infty \\
\stackrel{\infty}{\infty} \\
\sim\end{array}$ & 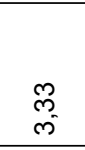 & $\begin{array}{l}\infty \\
\stackrel{\infty}{\infty} \\
\sim\end{array}$ & 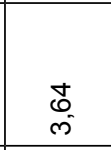 & $\begin{array}{l}0 \\
\stackrel{N}{m} \\
\sim\end{array}$ & 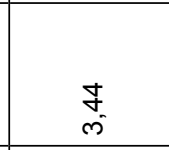 & 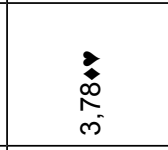 & $\begin{array}{l}\bullet \\
\ddot{\infty} \\
\oplus\end{array}$ \\
\hline & $\begin{array}{l}\frac{9}{0} \\
\frac{\pi}{0} \\
\frac{0}{0} \\
\frac{0}{E} \\
\bar{\Xi} \\
0\end{array}$ & 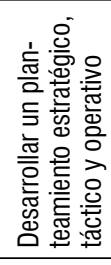 & 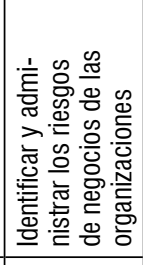 & 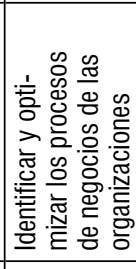 & 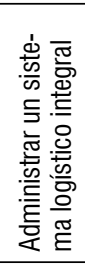 & 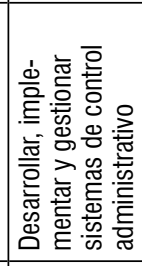 & 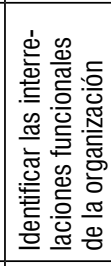 & 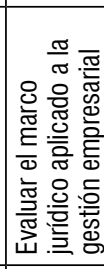 & 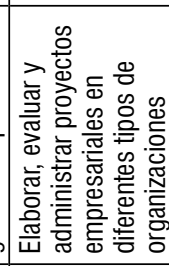 & 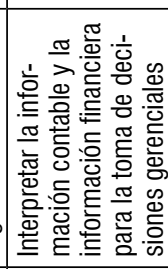 & 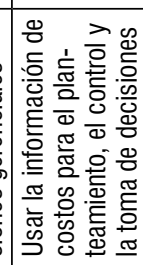 \\
\hline & * & - & $\sim$ & $\infty$ & $\nabla$ & ما & 0 & $\Lambda$ & $\infty$ & $\infty$ & 으 \\
\hline
\end{tabular}


IMPORTANCIA Y COINCIDENCIA DE COMPETENCIAS PARA EGRESADOS DE ADMINISTRACIÓN Y EMPLEADORES:

UN ENFOQUE POR REGIONES DE COLOMBIA

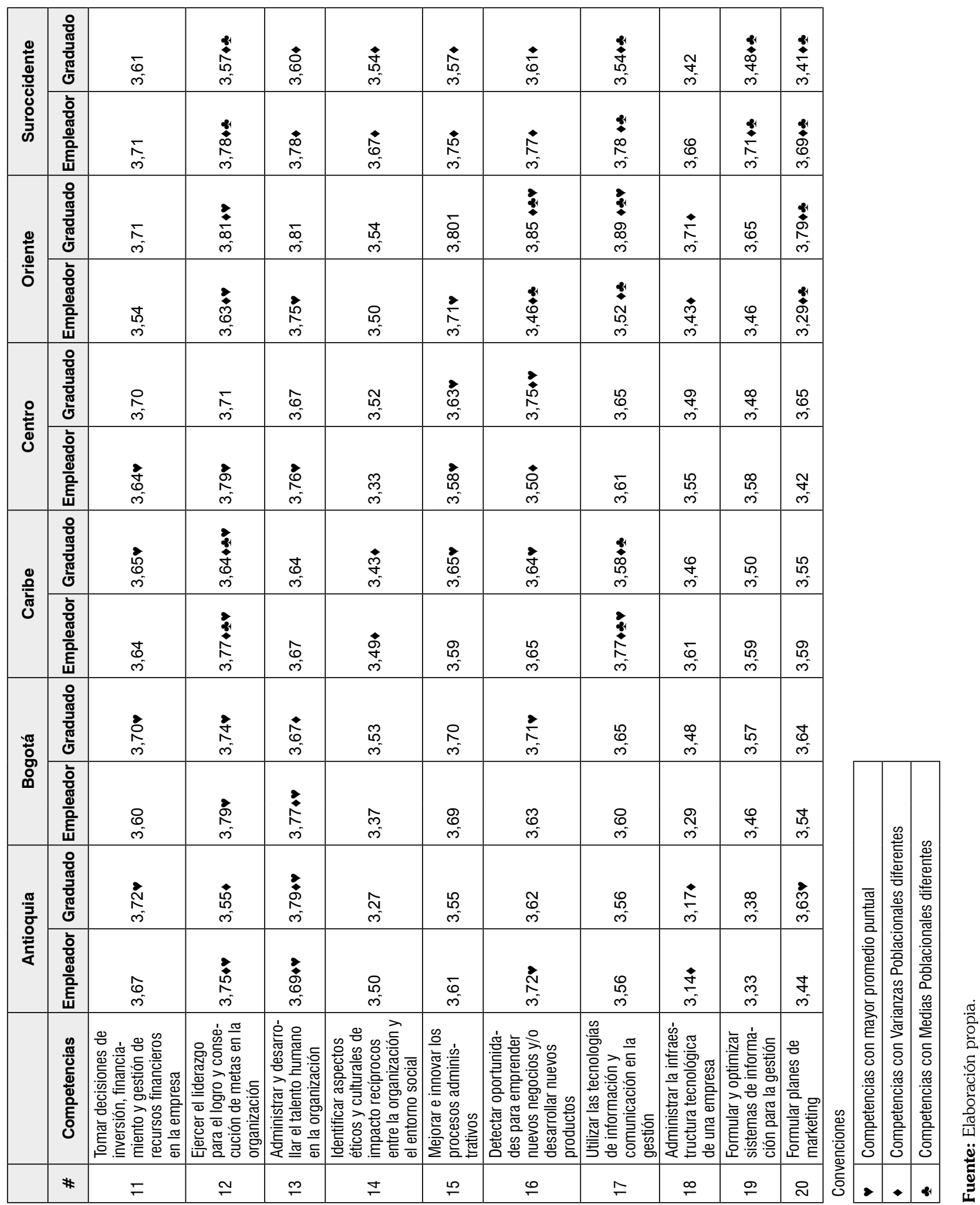


El promedio de importancia puntual asociado en cada grupo a cada una de las competencias específicas por región geográfica se observa en la Tabla 6 , las competencias con corazón ( $\boldsymbol{\vee}$ ) son las cinco competencias con mayor importancia puntual por grupo y las identificadas con trébol (\$) son las que presentan diferente media poblacional de acuerdo a la prueba de hipótesis.

En Antioquía las competencias que coinciden son 1 y 13 (2 de 5). Los promedios oscilan entre [3,14; $3,78]$ y $[3,17 ; 3,79]$. De acuerdo a la docimasia de igualdad de medias poblacionales para las $20 \mathrm{com}^{-}$ petencias no es significativa la diferencia para los dos grupos.

En Bogotá coinciden en la 1 y 12 (2 de 5); los promedios oscilan entre: $[3,54 ; 3,83]$ y $[3,43 ; 3,75]$. Las competencias para las que sí es significativa la diferencia de medias son 5 y 7 .

En el Caribe coinciden en la 12 (1 de 5). Los promedios oscilan entre $[3,4 ; 3,8]$ y $[3,4 ; 3,67]$ respectivamente para empleadores y graduados. Las competencias con diferencias de medias significativas son: 1,12 y 17 .

En el Centro, son comunes en la 9 (1 de 5). Los promedios para empleadores y graduados oscilan entre $[3,33 ; 3,79]$ y $[3,39 ; 3,79]$ respectivamente. Solo para la 10 es significativa la diferencia de medias.

En el Oriente coinciden en la 2 y 12 (2 de 5). Los promedios oscilan dentro de $[3,29 ; 3,75]$ empleadores y $[3,48 ; 3,88]$ graduados. La diferencia de medias es significativa en las competencias: $(7,8$, $9,10,16,17$ y 20$)$.

En el Suroccidente coinciden en la 1, 2 y 3 (3 de 5). Los promedios oscilan para empleadores y graduados dentro de $[3,63 ; 3,89]$ y $[3,41 ; 3,71]$ respectivamente. La diferencia de medias es significativa en las competencias: $(1,2,3,5,6,9,10,12,17$, 19 y 20$)$.

Las competencias especificas que presentan mayor variabilidad en las seis regiones son: $1,10, y$
12 , en estas competencias es significativa la diferencia de las varianzas poblacionales en el $67 \%$ de las regiones. Las competencias 2, 3, 9, 13, 16 y 17 muestran variabilidad diferente en el $50 \%$ de las regiones. Las competencias 5, 7, 14, 18 y 20 son diferentes en el $33 \%$ de las regiones.

Las competencias específicas más importantes en las seis regiones son: desarrollar el pensamiento táctico y operativo (1); interpretar la información contable y la información financiera para la toma de decisiones gerenciales (9). En el $83 \%$ de las regiones, tienen como las más importantes: ejercer el liderazgo para el logro y consecución de metas en la organización (12); detectar oportunidades para emprender nuevos negocios y/o desarrollar nuevos productos (16). En el 67\% de las regiones se encuentran: identificar y administrar los riesgos de negocios en las organizaciones (2); tomar decisiones de inversión, financiamiento y gestión de recursos financieros en la empresa (11) y en el $50 \%$ de las regiones se encuentran: administrar y desarrollar el talento humano en las organizaciones (13); identificar y optimizar los procesos de negocios de las organizaciones (3); usar la información de costos para el planteamiento, el control y toma de decisiones (10) y mejorar e innovar los procesos administrativos (15).

\section{Conclusiones}

Como resultado de la investigación se encontró que en las diferentes regiones del país los empresarios $y$ graduados coincidieron en algunas de las 5 competencias más importantes tanto genéricas como específicas, a continuación se presentan para cada una de las regiones las competencias coincidentes. Cuando se encuentran estas coincidencias en las competencias, indica que ambos están de acuerdo en cuáles son los perfiles que se deben manejar en cada región.

En Antioquia las competencias coincidentes son: capacidad para tomar decisiones, compromiso ético, compromiso con la calidad, desarrollar un planteamiento estratégico, táctico y operativo, administrar y desarrollar el talento humano en la organización (esto es el $50 \%$ de las competencias 
requeridas. Esto implica por lo tanto que el perfil laboral esperado para un candidato a un empleo es poseer todos los elementos necesarios y suficientes para tomar decisiones en cualquiera de los escenarios de la organización, que sea capaz de desarrollar planteamientos estratégicos en cualquiera de las áreas de la empresa, que sea oportuno y objetivo en lo táctico y operativo, una persona convencida que el talento humano al interior de la organización es vital no solo para la supervivencia sino también para su crecimiento y por lo tanto se comprometa con el fortalecimiento de esta área, y que crea que la calidad es uno de los diferenciadores positivos de las empresas y que por lo tanto hay que trabajar permanentemente porque la calidad se construye día a día. Dado que es una persona comprometida con la ética, lo anterior lo trabaja dentro de un marco conceptual ético desde la perspectiva teórica y práctica. Los empleadores solicitan también estas competencias: habilidades para tomar decisiones; estrategias para emprender nuevos negocios, soportados en el liderazgo para lograr las metas de la organización y en la información contable financiera, así como habilidades para solucionar problemas y trabajar en equipo, estas competencias aunque valiosas para los graduados no las incluyeron dentro de las cinco más importantes.

En Bogotá las competencias coincidentes son: capacidad para identificar, planear y resolver problemas, desarrollar un planteamiento estratégico, táctico y operativo, ejercer el liderazgo para el logro y consecución de metas en la organización, compromiso con la calidad (esto representa solo el $40 \%$ de las competencias requeridas). Esto implica por lo tanto que el perfil laboral que se espera para un candidato a un empleo, es que tenga las características apropiadas para identificar rápida y oportunamente cualquier problema que afecte a la organización, por lo tanto que sea capaz de plantear las estrategias y planear las acciones a los problemas concretos $y$ así garantizar la sostenibilidad de la empresa en un entorno altamente competitivo donde la calidad es una de las variables que marca el diferencial, por ello el candidato debe estar realmente comprometido con ella. Con sus dotes de líder debe conducir a todas las fuerzas vivas de la empresa para que esta alcance todas las metas que se ha propuesto.
Las otras competencias que requiere el empleador aunque valiosas para el egresado, no las incluyeron dentro de las cinco más importantes, son: identificar y administrar el riesgo, soportado en procesos óptimos, en la calidad y desarrollo del recurso humano; también pide responsabilidad social; abstracción, análisis y sintesis y con una buena habilidad de comunicación.

En el Caribe las competencias coincidentes son: capacidad de aplicar los conocimientos en la práctica, capacidad para tomar decisiones, compromiso con la calidad, ejercer el liderazgo para el logro y consecución de metas en la organización (esto es el $40 \%$ de las competencias requeridas). Esto implica por lo tanto que el perfil laboral que se espera para un candidato a un empleo es que posea todos los elementos necesarios y suficientes para tomar decisiones en cualquiera de los escenarios de las organizaciones. Se espera que con sus dotes de líder comprometa a toda la organización a enriquecerse con sus planteamientos novedosos, con sus teorías de punta, en procura de alcanzar una alta calidad que es una de las metas de la organización. Otras competencias requeridas por los empleadores aunque valiosas para los graduados no las incluyeron dentro de las cinco más importantes, son: planteamientos tácticos y operativos; optimización de los procesos; soportándose en la información de costos; apoyado en la tecnología de la información y comunicación; ser organizado con el tiempo y ser ético.

Las competencias coincidentes en el Centro son: interpretar la información contable y la información financiera para la toma de decisiones gerenciales, compromiso ético (esto es el $20 \%$ de las competencias requeridas). Esto implica por lo tanto que el perfil laboral que se espera para un candidato a un empleo debe tener una formación sólida en el área contable y en el área financiera, de forma tal, que cuando la organización requiera tomar decisiones a un alto nivel en estos campos, él debe ser quien garantiza el éxito y él quien asume las responsabilidades dentro un marco ético tanto desde el punto de vista teórico y práctico. Otras competencias pedidas por empleadores que aunque valiosas para los graduados no las incluyeron en las cinco más importantes son: control administrativo; liderazgo 
para la consecución de las metas; toma de decisiones de inversión; financiación y gestión del recurso financiero; administración y desarrollo del recurso humano; capacidad creativa; capacidad de toma decisiones; saber trabajar en equipo; y con compromiso con la calidad.

Las competencias coincidentes en el Oriente son: capacidad de aprender y actualizarse permanentemente, capacidad para tomar decisiones, capacidad de trabajo en equipo, identificar y administrar el riesgo de negocios de las organizaciones, ejercer el liderazgo para el logro y consecución de metas en la organización (esto es el $50 \%$ de las competencias para la empleabilidad requeridas por los empleadores). Esto implica por lo tanto que el perfil laboral que se espera para un candidato a un empleo es que no solo sea capaz de trabajar en equipo sino también que organice buenos equipos de trabajo y que con sus dotes de líder induzca a sus grupos a estar al tanto de las nuevas teorías y de las tecnologías de punta y así las decisiones que se tomen en cualquiera de las áreas de la empresa redunde en la mejora continua de la organización y en el alcance de las metas propuestas y que con la ayuda de esa actualización permanente con la que debe comprometerse, se maneje el equilibrio en la administración de los riesgos porque el asumir un riesgo es prepararse para un logro o un fracaso, por lo tanto, que él sea capaz de evaluar el fracaso como oportunidad de aprendizaje, y de prever que el fracaso no lesione irreversiblemente a la empresa. Las otras competencias requeridas por empleadores aunque valiosas para los graduados no las incluyeron dentro de las cinco más importantes, son: desarrollar un pensamiento táctico y operativo; administrar y desarrollar el talento humano en la organización; mejorar e innovar procesos administrativos; identificar, planear y resolver problemas y compromiso ético.

Las competencias coincidentes en el Suroccidente son: capacidad para tomar decisiones, compromiso ético y desarrollar planteamiento estratégico, táctico y operativo, identificar y administrar el riesgo de negocios de las organizaciones, identificar y optimizar los procesos de negocios de las organizaciones (esto es el $50 \%$ de las competencias pedidas por empleadores). Esto implica por lo tanto que el perfil laboral que se espera para un candidato a un empleo es que posea todos los elementos necesarios y suficientes para tomar decisiones en cualquiera de los escenarios de la organización, que sea capaz de desarrollar planteamientos estratégicos en cualquiera de las áreas de la empresa, que sea oportuno, objetivo en lo táctico y operativo. Que sea capaz de manejar el equilibrio en la administración de los riesgos porque el asumir un riesgo es prepararse para un logro o un fracaso, por lo tanto que él evalúe el fracaso como oportunidad de aprendizaje, y que prevea que el fracaso no lesione irreversiblemente a la empresa, que tenga la táctica para abordar los procesos de los negocios para administrarlos, mejorarlos, cambiarlos, si es necesario y así tomar las decisiones en cuanto a negocios se refiere, de una manera clara, oportuna y pertinente; como es una persona comprometida con la ética, no sólo desde el punto de vista teórico sino también en la realidad, todas estas actividades las desarrollará dentro del marco de la ética. Las otras competencias requeridas por los empleadores, aunque valiosas para los graduados no las incluyeron en las cinco más importantes, son: desarrollar e implementar y gestionar sistemas de control administrativo e interpretar la información contable financiera para la toma de decisiones gerenciales.

Como se observa en las seis regiones, Antioquia, Oriente y Suroccidente coinciden con el 50\% de las cinco competencias específicas y cinco genéricas más importantes solicitadas para ser el empleado. Es importante resaltar que el promedio de importancia asociado por empleadores y egresados no es diferente en la mayoría de las competencias, pero no están en el mismo orden. Bogotá y Caribe solo coinciden en el $40 \%$ y en el Centro solo coinciden en el $20 \%$. Esto no indica que las competencias importantes para los empleadores no lo son para graduados, hay otras que estos ven más relevantes. Los graduados están más inclinados a los nuevos negocios y productos así como en la diversificación de los negocios, se muestran como una generación más emprendedora. En el Suroccidente y Centro a los egresados también les preocupa el riesgo de los negocios. Destacan como coincidentes en las competencias genéricas de esta investigación y de la revisión de la literatura: capacidad para identificar, 
planear y resolver problemas, capacidad para tomar decisiones y compromiso ético.

Esto muestra una brecha en lo solicitado por los empleadores y lo considerado importante para los egresados, principalmente en las competencias específicas. Para próximos estudios sería importante definir si los requerimientos del empleador se han quedado en los requerimientos actuales y la visión de los egresados está a la vanguardia de las necesidades de la empresa en un futuro cercano, lo cual indicaría que las instituciones de educación superior están preparando gente para el cambio.

Todo esto puede indicar que las instituciones educativas estarían formando una generación con una visión más independiente, más emprendedora, lo que implicaría generar nuevas fuentes de trabajo. De acuerdo a la revisión de la literatura las empresas buscan gerentes formados en competencias genéricas sin desconocer las habilidades propias de la disciplina. Los ambientes cambiantes obligan a que las competencias también evolucionen lo que deja opciones de investigación a futuro en esta temática.

Estos resultados también pueden ser insumos susceptibles de análisis en la construcción de los currículos; no sólo desde la perspectiva de las expectativas del aparato productivo sino también desde la necesidad de elaborar currículos más homologables entre las diferentes regiones y de esta forma hacer más amigable el tránsito de los estudiantes entre los diferentes programas de administración.

\section{Referencias}

Aguilar-Joyas, J. (2015). Competencias específicas Tuning en programas de administración: Colombia y su región Suroccidente. En: Revista Contexto, Universidad La Gran Colombia, 4: 111-117.

Asociación Colombiana de Facultades de Administración - ASCOLFA \& Grupo Interinstitucional de Investigación de Competencias en Administración - GRIICA. (2010). Las competencias de los administradores en Colombia a la luz del proyecto Tuning América Latina 2008-2010. Bogotá - Colombia: ASCOLFA.

Beach, D. (1982). A Training Program to Improve Work Habits, Attitudes, and Values. En: Journal of Epsilon Pi Tau, 8 (2): 69-74.

Beneitone, P., Esqueti, C., González, J., Marty, M., Siufi, G. \& Wagenaar, R. (2007). Reflexiones y perspectivas de la edu- cación superior en América Latina. Informe final-proyecto Tuning-América Latina 2004-2007. Universidad de Deusto-Universidad de Groningen.

Boyatzis, R. (1982). The Competent Manager: A Model for Effective Performance. New York: John Wiley and Sons.

Bunk, G. (1994). La transmisión de las competencias en la formación y perfeccionamiento profesionales de la RFA. En: Revista Europea Formación Profesional, Organización de Estados Iberoamericanos - OEI, 1: 8-14.

Byrne, J. (2014). Why the MBA has become the most popular master's degree in the U.S. En: Poets\&Quants. Disponible: http://fortune.com/2014/05/31/mba-popular-mastersdegree/

Castrillón, J; Cabeza L \& Lombana, J. (2015). Competencias más importantes para la disciplina administrativa en Colombia. En: Contaduría y Administración, Universidad Autónoma de México, 60 (04): 776-795.

Chou, Y. (1987). Análisis Estadístico. México: Nueva Editorial Interamericana.

Cotton, K. (1993). Developing Employability Skills. En: School Improvement Research Series. (SIRS), CloseUp \#15.

Daza, A.; Charris, A. \& Viloria, J. (2015). Competencias específicas de los administradores como: factor de desarrollo. En: Dimensión Empresarial, Universidad Autónoma del Caribe, 13 (2): 275-292.

Declaración de Bologna (1988) Magna Charta Universitatum. Disponible: http://www.magna-charta.org/resources/files/ the-magna-charta/spanish

Dishman, L. (2016). These Are The Biggest Skills That New Graduates Lack. En: The Fast Company Disponible: http:// www.fastcompany.com/3059940/the-future-of-work/theseare-the-biggest-skills-that-new-graduates-lack

Ducci, M. (1997). El enfoque de competencia laboral en la perspectiva internacional. Seminario internacional sobre formación basada en competencia laboral: situación actual y perspectivas, Guanajuato, México.

Esquetini, C. (editor) (2013). Educación Superior en América Latina: reflexiones y perspectivas en Administración. Bilbao: Tuning Academy. Disponible: http://tuningacademy.org/ wp-content/uploads/2014/02/RefAdministration_LA_SP.pdf

Fitó-Bertran, A.; Martínez, M. \& Moya S. (2014). El perfil competencial de los graduados de Administración y Dirección de Empresas en línea: una visión desde el mercado de trabajo. En: Revista de Universidad y Sociedad del Conocimiento, Universitat Oberta de Catalunya y University of New England, 11, (2): 13-26.

Gallart, M. (1998). Competitividad, redes productivas y competencias laborales. Montevideo: CINTERFOR, OIT.

González, J y Wagenaar, R. (2003). Tuning Educational Structures in Europe. Informe Final. Fase Uno. Bilbao: España: Universidad de Deusto y Universidad de Groningen.

González, J. \& Wagenaar, R. (2006) Tuning Educational Structures in Europe II: La Contribución de las Universidades al Proceso de Bologna. Universidad de Groningen \& Universidad de Deusto. Disponible: http://www.deusto-publicaciones.es/ deusto/pdfs/tuning/tuning04.pdf 
Gonzci, A. \& Athanasou, J. (1994). Instrumentación de la educación basada en competencias. Perspectivas de la teoría y práctica en Australia. En: Competencia laboral y educación basada en normas de competencias, Limusa, 19: 265-288.

Hoffmann, T. (1999). The meanings of competency. En: Journal of European Industrial Training, Emerald Insight, 23 (6): 275-285.

Jackson, D. (2013). Business graduate employability - where are we going wrong?. En: Higher Education Research \& Development, Taylor \& Francis, 32 (5): 776-790.

Kavanagh M. H. \& Drennan L. (2008). What skills and attributes does an accounting graduate need? Evidence from student perceptions and employer expectations. En: Accounting \& Finance, Affanz, 48 (2): 279-300.

Koshansky, J. (1998). El Sistema de Competencias. En: Training Development Digest. Madrid. Disponible: http://www.oitcinterfor.org/p\%C3\%A1gina-libro/definiciones-algunos-expertos

Malpica, M. (2000). El punto de vista pedagógico. Competencia laboral y educación basada en normas de competencia. México, D.F.: Limusa, SEP, CNCCL y CONALEP.

Martensen, A. \& Grønholdt, L, (2009). Quality in higher education: linking graduates' competencies and employers' needs. En: International Journal of Quality and Service Sciences, Emerald Insight, 1 (1): 67 - 77.

McClelland, D. (1973) Testing for Competence rather than for Intelligence. En: American Psychologist, APA, 28 (1): 1-14.

Mertens, L. (1997). Sistemas de competencia laboral: surgimiento y modelos. Formación basada en competencia laboral: Situación actual y perspectivas. 1, Guanajuato, México, CINTERFOR. Disponible: http://www.oei.es/etp/ formacion_basada_competencia_laboral.pdf

Ministerio de Educación Nacional - MEN (2008-2013). Encuesta Observatorio Laboral para la Educación. Disponible: http://www.graduadoscolombia.edu.co/html/1732/w3article-156441.html

Ministerio de Educación Nacional - MEN (2010) Aportes para la Construcción de Currículos Pertinentes. Articulación de la Educación con el Mundo Productivo. Serie Guías No.21.

Ministerio de Educación Nacional. (s.f.) (recuperado en mayo de 2016) Observatorio Laboral para la Educación. Disponible: http://www.graduadoscolombia.edu.co/html/1732/w3channel.html

Mole, V., Dawson, S., Winstanley, D. \& Sherval, J. (1993). Researching Managerial Competences. British Academy of Management Annual Conference, Milton Keynes.

OECD. (2004). The Definition and Selection of Key Competencies. Paris: OECD. Disponible: https://www.oecd.org/ pisa/35070367.pdf

Paadi, K. (2014). Perceptions on Employability Skills Necessary to Enhance Human Resource Management Graduates Prospects of Securing A Relevant Place in The Labour Market. En: European Scientific Journal, European Scientific Institute. August 2014, Special Edition, 129-143.

Parra-Alviz, M.; Ramírez, E. \& Montero G. (2015). Análisis de las Competencias en Programas Académicos de Administración de Empresas: Estudio Empírico en la Universidad del Tolima. En: Revista de Economía \& Administración, Universidad Autónoma de Occidente. 12 (2): 47-61.

Raybould, J. \& Sheedy, V. (2005). Are graduates equipped with the right skills in the employability stakes?. En: Industrial and Commercial Training, Emerald Insight, 37 (5): 259 - 263.

Ruiz, M. (2005). Enfoque metodológico para la formación de competencias desde el ámbito educativo: Cómo concretar una alternativa para la relevancia educativa. En: Revista Enunciación, Universidad Distrital de Colombia, 10 (1): 85-93.

The Economist. (2001). What companies want from an MBA graduate. En: The Economist. Disponible: http://www. economist.com/node/560852

Tuning Academy (s.f.). (recuperado en mayo de 2016) Projects related to subject area "Business Administration. Disponible: http://tuningacademy.org/category/thematic-areas/businessadministration/?lang $=e n$

UNESCO. (2011). Enfoque por Competencias. Oficina Internacional de Educación. Disponible: http://www.ibe.unesco. org/es/comunidades/comunidad-de-practica-cop/enfoquepor-competencias.html

Universia. (2011). Lo que las empresas quieren de un recién egresado. En: Revista Universia. Disponible: http://noticias. universia.edu.ve/en-portada/noticia/2011/06/03/833247/ empresas-quieren-recien-egresado.html

Valero, G. (2011). Las competencias de los administradores en Colombia a la luz del Proyecto Tuning - Caso Capitulo Oriente. En: Puente Revista Científica, UPB-Bucaramanga, 5 (1): $73-82$

Victorino, L. \& Medina, G. (2008). Educación basada en competencias y el proyecto Tuning en Europa y Latinoamérica. En: Ide@s, CONCYTEG, 3 (39): 97-114.

Vygotsky, L. (1979). El desarrollo de los procesos psicológicos superiores. Barcelona: Crítica.

Weber, M. (1971). Économie et société. París: Plon. De la versión alemana Wirtschaft und Gesellschaft (1921).

Wentling, R. (1987). Employability Skills: The Role of Business Education. En: Journal of Education for Business. Taylor \& Francis, 62 (7): 313-317.

Willis, T. \& Taylor, A. (1999). Total quality management and higher education: the employers' perspective. En: Total Quality Management, Routledge, 10 (7): 997-1007.

Wilton, N. (2011) Do employability skills really matter in the UK graduate labour market? The case of business and management graduates. En: Work, employment and society, Sage Ed., 25 (1): 85-100.

Wilton, N. (2012) The impact of work placements on skills development and career outcomes for business and management graduates. En: Studies in Higher Education, Routledge, 37 (5): 603-620.

Zinser, R. (2003). Developing career and employability skills: a US case study. En: Education + Training, Emerald Insight, 45 (7): $402-410$. 